\section{Análisis de la ocurrencia de la Mortalidad Materna en la Provincia de Santa Fe - Argentina, a propósito de una intervención}

\section{Occurrence analysis of Maternal Mortality in the Province of Santa Fe - Argentina, due to an intervention}

\section{Resumen}

Introducción: La Mortalidad Materna (MM) muestra diferencias en las condiciones de salud y de vida de las poblaciones, constituyéndose en un buen indicador socio-económico, así como de la cobertura y calidad de los servicios de atención a la mujer en edad fértil. Las estadísticas oficiales de salud no revelan la magnitud del problema de la MM debido al subregistro que posee esta causa de muerte. Dicha falencia se señala especialmente para los países subdesarrollados. Se estima que la República Argentina no escapa al fenómeno del subregistro. La provincia de Santa Fe en el año 1994 decidió incorporar a su Certificado de Defunción un ITEM específico para la causa Muerte Materna con el fin de corregir el subregistro. Objetivos: El objetivo principal de este estudio fue evaluar los cambios que registró la Razón de Mortalidad Materna (RMM) en el período 1988-2001, como consecuencia de la introducción del "ITEM 33" referido a la Muerte Materna, en el Certificado de Defunción de la Provincia de Santa Fe a partir del año 1995. Material y Métodos: Se revisó la bibliografía respecto del subregistro de la MM. Se estudió la ocurrencia de la MM en la Provincia de Santa Fe en el período 1988-2001. Se analizaron los cambios producidos por la presencia del "ITEM 33" en el Certificado de Defunción de la Provincia de Santa fe en el período 1995-2001. Resultados: Se observó un aumento en las cifras absolutas de MM y por ende de las razones de MM (41\%) en el período 1995-2001, a diferencia de la tendencia descendente de este indicador tanto a nivel nacional como a nivel provincial en el período anterior (pre “ITEM 33”). Se observó además, una modificación en la distribución de las muertes respecto a su incidencia en los grupos etáreos. Otro dato de interés es que se modificó la frecuencia de las patologías que originaron la muerte (causa de muerte) siendo el Aborto la causa más frecuente, a diferencia de los períodos anteriores donde aparecía la Hipertensión Arterial ocupando el primer lugar. Conclusión: La introducción del "ITEM 33" en el Certificado de Defunción de la Provincia de Santa Fe produjo modificaciones importantes en los datos sobre MM. Estas modificaciones pueden ser atribuidas a la disminución del subregistro, ya que no se encuentran razones valederas para pensar en un aumento real en la ocurrencia de muertes maternas. Por lo cual se concluye que: este instrumento de recolección de datos permitiría corregir el subregistro y obtener datos de mayor calidad que, en consecuencia, posibiliten la implementación de políticas y programas de salud basados en datos que reflejen la verdadera situación sanitaria.

Palabras Claves: Mortalidad Materna. Subregistro. Tasa de Mortalidad Materna. Razón de Mortalidad Materna. Certificado de Defunción. Causa de Muerte. 
Abstract

Introduction: Maternal Mortality (MM) shows differences in population health and life conditions, therefore it becomes not only a good socioeconomic indicator but also an indicator of health service providers and health care service quality in the assistance to women at reproductive age. Official Health Statistics do not reveal the size of MM problem due to the under-report that has this death cause. This deficiency or lack of data is highly marked especially in underdeveloped countries. It has been estimated that Argentina is not out of this under-report problem. In 1994, the Province of Santa Fe decided to add a specific ITEM to the Death Certificate for Maternal Death cause with the aim of amending the under-report. Objectives: The main objective of this study was to evaluate changes that Maternal Mortality Ratio (MMR) registered between 1988 and 2001 as a consequence of adding the "33 ITEM" regarding Maternal Death to the Death Certificate of the Province of Santa Fe from 1995 on. Material and Methods: Bibliography was reviewed as regards the MM under-report. The MM occurrence in the Province of Santa Fe was studied from 1988 to 2001. Changes produced by the 33 ITEM appearance in the Death Certificate of the Province of Santa Fe during the period 1995 - 2001 were analyzed. Results: It was seen an increase in the MM absolute figures and therefore, an increase of MM ratios (41\%) from 1995 to 2001, unlike the lowering trend of this indicator both at national level and at provincial level in the previous period (pre "33 Item"). Besides, it was seen a modification in death distribution in relation to its incidence among diferent age groups. Another important detail is that frequency of pathologies leading to death (death cause) was modified, being Abortion the most frequent cause unlike the previous periods in which Hypertension was first in the rank. Conclusion: The "33 ITEM" addition to Death Certificate of the Province of Santa Fe brought about important MM data modifications. These modifications should be attributed to the decrease of under - reporting since there is no valid reason to think of a real increase in maternal death occurrence. Thus, as a conclusion, this instrument for collecting data allows to amend the under-report and obtain higher quality data, which consequently bring the possibility of setting up health policy and programmes based on data showing the real health situation.

Key Words: Maternal Mortality. Underreport. Maternal Mortality Rate. Maternal Mortality Ratio. Death Certificate. Death Cause.

\section{Introducción}

La mortalidad de mujeres por causas derivadas del proceso reproductivo refleja no solo una pérdida biológica y psicosocial en términos de muertes ocurridas, sino también el grado de severidad, de un costo y una medida predictiva, de la calidad de vida ylas perspectivas futuras, para aquellas que sobreviven. También muestra diferencias en las condiciones de salud y de vida, constituyéndose en un buen indicador socio-económico, así como de la cobertura y calidad de los servicios de atención a la mujer en edad fértil ${ }^{1}$.

La muerte de una mujer por motivos de la maternidad debe ser entendida como expresión de la relativa desventaja que experimentan importantes sectores femeninos de la región en el logro de sus derechos fundamentales y de la maternidad deseada .

Hasta hace muy recientemente la Mortalidad Materna (MM) era considerada como un problema de relativa poca importancia para la salud pública en la gran mayoría de los países de Latinoamérica y el Caribe.

Un hecho importante a tener en cuenta, es que las estadísticas oficiales de salud no revelan la magnitud del problema de la MM debido al subregistro que posee este indicador. Esta falencia se señala especialmente para los países subdesarrollados. En países como Egipto, India y Turquía, las cifras reales de MM se ubican entre un 25 y un $80 \%$ por encima de las cifras oficiales ${ }^{2}$.

Sin embargo los países desarrollados no están exentos de la problemática antes mencionada, tal como lo demuestran las investigaciones de Benedetti en Washington entre los años 1977 y $1981^{3}$, y más recientemente Allen y col. ${ }^{4}$, quienes demostraron en una investigación realizada en la ciudad de New York (EE UU), un incremento porcentual de números absolutos de MM del $18 \%$ para el período estudiado.

Entre las causas que provocan esta situación se pueden mencionar: una incorrecta clasificación de enfermedades, la falta de una adecuada formación del médico en su etapa de formación de grado y pos grado, para el correcto llenado del Certificado de Defun- 
ción, cuando no el deliberado ocultamiento de la verdadera causa de muerte. Es decir, la "causa de muerte" declarada en el Certificado de Defunción, muchas veces no refleja que la misma sea materna como lo demostraron Puffer ${ }^{5}$ y otros autores ${ }^{6}$.

Diversos documentos oficiales de OPS, demuestran que las tasas oficiales de mortalidad por causas maternas son inferiores a su verdadera ocurrencia ${ }^{7,8}$. Laurenti ${ }^{9}$, mediante una corrección de datos oficiales, determinó que la mortalidad materna en San Pablo, Brasil, que oficialmente era de 50,2 por 100 mil nacidos vivos en el año 1986, pasó a ser de 99,7 por 100 mil o sea casi el doble en ese mismo año después de una revisión de los certificados de defunción.

Recientemente, el documento "Estrategia Regional para la Reducción de la Mortalidad y Morbilidad Maternas", presentado en la 26va. Conferencia Sanitaria Panamericana de Septiembre de 2002, en su página seis confirma "la subnotificación de las estadísticas oficiales es un grave motivo de preocupación.”10.

La República Argentina no escapa a este fenómeno del subregistro, sin embargo muy pocos estudios han sido publicados demostrando esta problemática. Entre ellos se puede citar al estudio de Althabe, Vinacur y col. realizado en Capital Federal, publicado por el Ministerio de Salud de la Nación en el año $1987^{11}$, y al realizado por el Ministerio de Salud de la Provincia de Córdoba en el año $1991^{12}$. A los que cabe agregar, aunque reconociendo el corto período de estudio, la cohorte publicada por Bloch y col. en los cuadernos Medico-sociales de Rosario, donde se evidencia un importante subregistro ${ }^{13,14}$.

En el citado estudio de Althabe y Vinacur, ya en el año 87 comentan: "Como ya se señaló, las dificultades en la recolección de los datos a partir de la certificación de la causa de muerte, harían suponer que esta tasa subestima la magnitud del problema...”

Y agregan más adelante: “...Con respecto a este último tema y, a manera de ejemplo, parece útil señalar que la revisión de los datos de mortalidad en el "Anuario de Estadísticas Vitales -Información Básica 1980-1981- pu- blicado por el Programa Nacional de Estadísticas de Salud, muestran que la frecuencia de muertes por septicemia no es diferente entre sexos hasta los quince años. A partir de esta edad y hasta los cuarenta y cinco años es dos o tres veces más frecuente en mujeres que en hombres y luego vuelven a igualarse. Es posible que una parte importante de estas muertes por septicemia, estén vinculadas al proceso reproductivo y por lo tanto inadecuadamente consignadas, dado que no se incluyen como muertes maternas".

\section{Importancia del tema}

La importancia del tema radica en que la Mortalidad Materna es una tragedia que en su mayoría es evitable ${ }^{15}$. Sus causas principales son bien conocidas y la información y tecnologías necesarias para lograr su reducción están a nuestro alcance.

La muerte de mujeres jóvenes y sanas, que ocurre durante el embarazo, parto o puerperio, tiene consecuencias profundamente negativas también para sus familias, $y$ otros miembros de la comunidad que estén relacionados con ellas.

Cuando estas muertes se multiplican o se mantienen en forma sostenida en el tiempo, representan un problema de salud pública y sobre todo un grave problema ético que exige una intervención urgente.

La MM es un problema que aún se mantiene vigente en grandes regiones del mundo. Se estima que 600.000 mujeres en todo el mundo mueren cada año, durante el embarazo, el parto o el puerperio, por causas relacionadas o agravadas por el embarazo ${ }^{16}$.

Las muertes maternas están directamente relacionadas con el subdesarrollo a punto tal que datos estimados por la Organización Mundial de la Salud muestran que el $99 \%$ de las muertes maternas ocurren en los países en subdesarrollo y solo el 1\%, ocurren en Europa, Norteamérica, Australia y Nueva Zelanda $^{17}$.

En la República Argentina se ha estimado que la TMM experimenta un subregistro que según las diferentes investigaciones oscila entre un 40 y un $70 \%^{18,19}$. En el informe 
"Progreso de las Naciones" UNICEF-OMS del año 1996, los datos correspondientes a nuestro país duplican las estadísticas oficiales, siendo concordantes estas cifras con las investigaciones antes señaladas.

Otra característica importante es que su ocurrencia varía ampliamente entre las regiones del país, reflejando grandes diferencias en la disponibilidad y calidad de cuidados obstétricos. Esta marcada variación, se observa al analizar subgrupos de población, con 1 muerte materna cada 794 nacimientos en la provincia de Corrientes, hasta 1 muerte cada 10.146 nacimientos en Ciudad de Buenos Aires $^{20}$.

El porcentaje de descenso de este indicador en el período 1970-1981 fue del $50 \%$, pasando de 140.3 a 70.0 cada 100.000 nacidos vivos. Desde el año 1980 hasta 1999 la Razón de Mortalidad Materna Nacional continuó disminuyendo llegando a 43 por cien mil, y manteniéndose relativamente estable desde entonces ${ }^{21}$.

\section{Objetivos del estudio}

Este estudio tuvo como objetivo general evaluar los cambios que registró la Razón de Mortalidad Materna en el período 1988-2001, como consecuencia de la introducción en el Certificado de Defunción de la Provincia de Santa Fe, del "ITEM 33" referido a la Muerte Materna, a partir del año 1995.

Se establecieron, además como objetivos específicos 1) Comparar los hallazgos en la estructura de las Muertes Maternas antes y después de la introducción en el Certificado de Defunción del “ITEM 33” y 2) Valorar los alcances de esta intervención, como método para solucionar el problema del subregistro de la Mortalidad Materna.

\section{Material y métodos}

Se utilizaron las definiciones de la Clasificación Estadística Internacional de Enfermedades y Problemas Relacionados con la Salud -Décima Revisión- Volumen I - OPS/ OMS (pág. 1169-1170), con las cuales trabaja la Dirección General de Estadística de la
Provincia de Santa Fe. Dependencia que publica en su anuario estadístico "Estadísticas Vitales, Series y Tendencias", las cifras oficiales de las cuales se recabaron los datos para esta investigación.

El indicador utilizado para estimar la Mortalidad Materna se debe denominar Razón de Mortalidad Materna (RMM). (muertes maternas sobre nacidos vivos, por factor de ampliación 100.000). Esta no es una "tasa o coeficiente" porque el denominador es el número de nacidos vivos, y el numerador el número de mujeres. Sin embargo es corriente el uso del término Tasas de Mortalidad Materna (TMM).

\section{Geosituación}

Los datos de este estudio corresponden a la provincia de Santa Fe, tercer distrito en importancia de la República Argentina. Posee una superficie total de $132.372 \mathrm{Km} 2$. Está dividida en 19 departamentos, y la habitan aproximadamente 2.800 .000 habitantes. El $82 \%$ de la población reside en centros urbanos y tiene una densidad poblacional promedio de 21,1 habitantes por $\mathrm{km} 2$.

Esta distribución no es homogénea, los departamentos del norte de la provincia, tienen una densidad media de $4 \mathrm{hab} / \mathrm{km} 2$, los del centro geográfico de $28 \mathrm{hab} / \mathrm{km} 2$, y los departamentos del sur provincial de $52 \mathrm{hab} /$ $\mathrm{km} 2$. Como ejemplo de esta desigual distribución poblacional mencionaremos que la ciudad de Rosario, perteneciente a la última región, posee una densidad poblacional de $4.560 \mathrm{hab} / \mathrm{km} 2$.

Teniendo en cuenta estas diferencias, se subdivide la provincia en tres "Zonas Geográficas” (Figura 1), las cuales se diferencian además, por sus características geográficas, por las condiciones de vida y de salud de la población.

La "Zona 1 o Norte" se encuentra ubicada en la Región Chaqueña, es la zona más extensa, solo reside en ella el $13 \%$ de la población la provincial. La "Zona 2 o Centro", preferentemente ganadera, posee el $28 \%$ de la población provincial. La "Zona 3 o Sur" pertenece a la pampa húmeda y gringa, con 


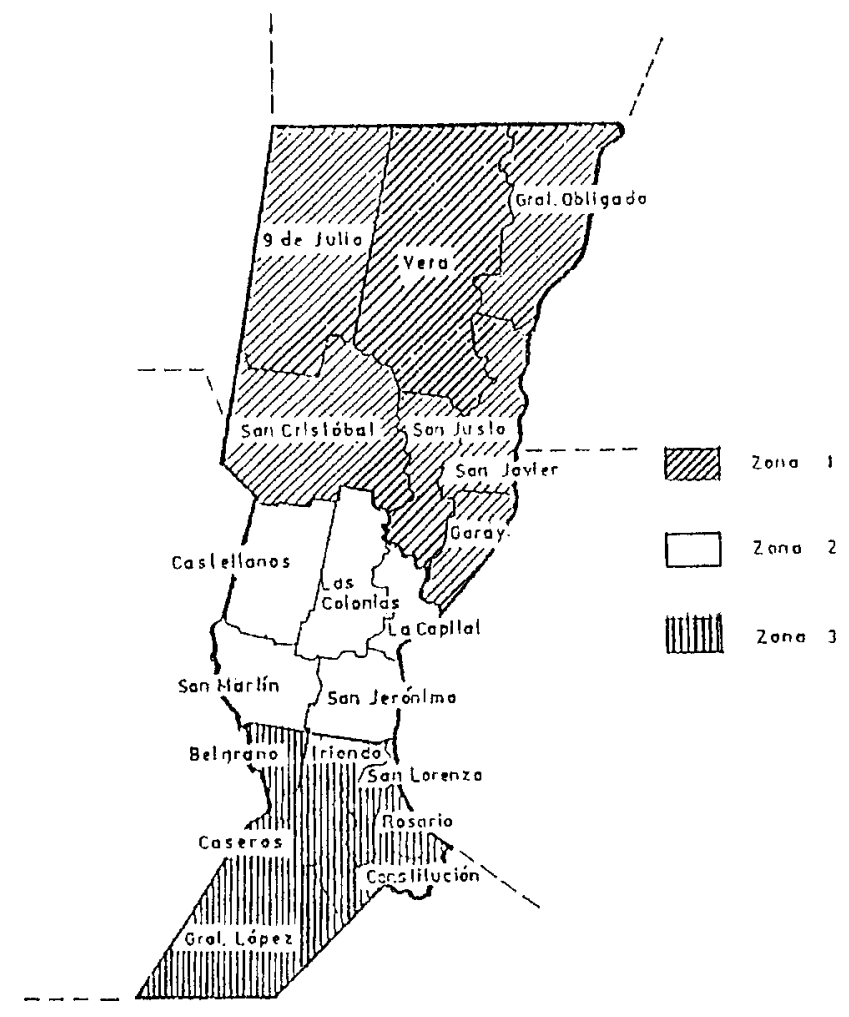

Figura 1 - Provincia de Santa Fe - Zonas Geográficas

Figure 1 - Santa Fe State-Geographic Zones

su potencial agricolo-ganadero. En ella se encuentran las ciudades y puertos más importantes de la provincia. En esta zona vive el $59 \%$ de la población de la provincia.

Cabeceras de las zonas geográficas señaladas, a saber: ciudad de Reconquista, Departamento Gral. Obligado (Zona Norte) con un solo efector; la ciudad de Santa Fe, Capital de la provincia (Zona Centro) con dos efectores; y la ciudad de Rosario (Zona Sur) con cinco efectores. Cada efector cuenta con Unidad de Terapia Intensiva Neonatal.

Otro dato importante es la preeminencia del sub-sector estatal como lugar de atención delos nacimientos. Ejemplo de ello es la información suministrada en el Boletín del Sistema de Estadísticas para la Salud; 2000 de la Secretaría de Salud Municipal de Rosario donde se señala que del total de nacimientos (16.570) el $67,12 \%$ de los mismos se produjeron en el sub-sector estatal; mientras que el $56 \%$ fueron en la orbita municipal y el $44 \%$ restante se realizó en dependencias provinciales.
Los datos sobre Mortalidad Materna en la provincia de Santa Fe comienzan a conocerse con la aparición de los primeros consolidados de datos estadísticos de salud, publicados por la Dirección General de Estadísticas del Ministerio de Salud provincial, con el nombre de "Estadísticas Vitales Provinciales" (total provincia) y del "Anuario Estadístico" del año 1980. No se encuentran publicaciones provinciales anteriores a esta fecha, que permitan el análisis de ocurrencia de las muertes maternas.

La publicación denominada "Estudios de Tendencias - Series cronológicas - Período 1.980-1.994" del anuario "Estadísticas Vitales" del Ministerio de Salud de la provincia deSanta Fe, revela una tendencia decreciente de la TMM para ese período (Gráfico. 1). Sin embargo las bajas tasas resultaron llamativas, estimándose que el subregistro podría ser su causa. El error en la certificación podría estar ocultando un importante número de muertes maternas. 


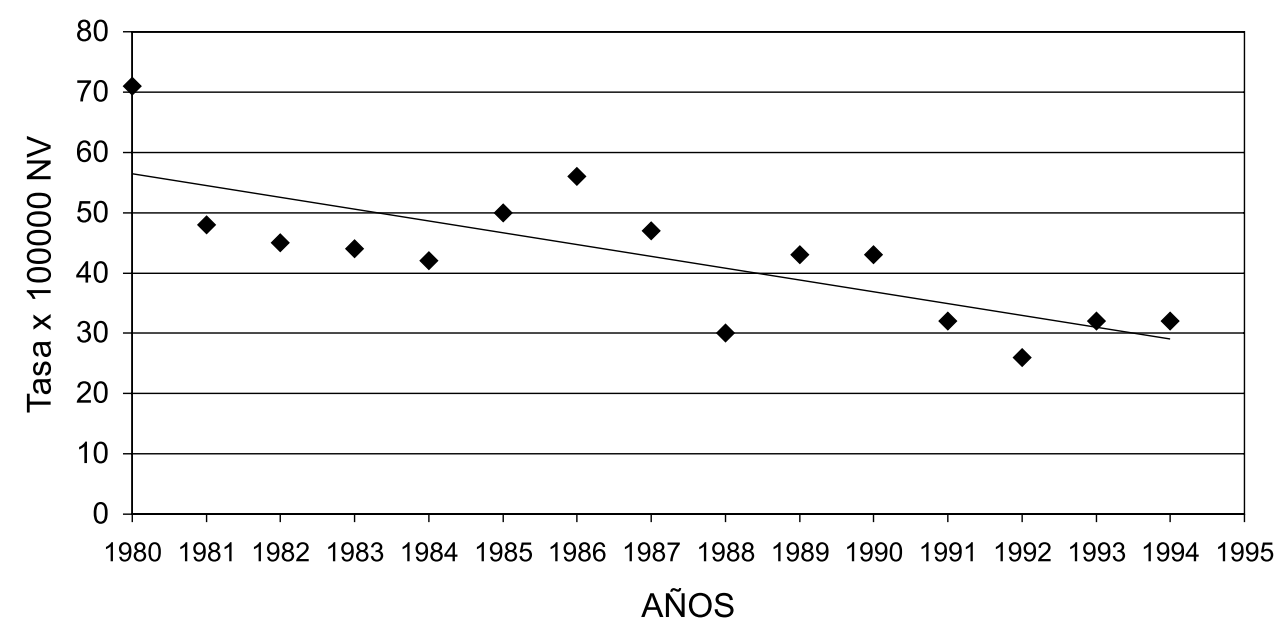

Fuente: Dirección General de Estadística - Ministerio de Salud Provincia de Santa Fe Source: Statistic Gral Direction - Health Ministry of Santa Fe State

Gráfico 1 - Tasa de Mortalidad Materna - Estudio de Tendencia Provincia de Santa Fe Período 1980-1994

Graphic 1 - Maternal Mortality Ratio - Trend Study - Santa Fe State - 1980-1994 Period

\section{Antecedentes del estudio}

En el año 1994 se realizó un estudio que analizó, mediante un muestreo al azar, la calidad del llenado de los Certificados de Defunción de la provincia de Santa Fe, donde se observó un deficiente y/o incorrecto llenado de los mismos por parte de los profesionales médicos, especialmente en elítem "Causa de Muerte", coincidiendo con lo observado por Torres de Quinteros y $\mathrm{col}^{22}$, en un estudio realizado en los años 1997/98.

Simultáneamente, se evaluaron los trabajos publicados referentes al subregistro de la Mortalidad Materna, tanto provinciales como nacionales, donde se pone de manifiesto la deficiente información y el subregistro que surge del análisis de los Certificados de Defunción para esa causa de muerte.

Teniendo en cuenta los antecedentes citados, se llevó a cabo en el mes de octubre del año 1994 en la ciudad de Rosario, provincia de Santa Fe, el "Primer Seminario Provincial sobre Mortalidad Materna”.

Del mismo participaron autoridades sanitarias nacionales, provinciales, municipales y universitarias expertos en la materia, donde se consensuaron importantes temas, tales como: adecuación del apartado "Causa de Muerte", creación del Comité de Vigilancia de la Mortalidad Materna, etc.

En dicho Seminario el autor de la presente investigación, presentó la propuesta de incorporación al Certificado de Defunción de un ITEM específico para la notificación de las muertes de mujeres en edad reproductiva. El mismo se denominó "ITEM 33" (Figura. 2). El “ITEM 33”, se ubica en el apartado "Certificación de Causa de Muerte" del Certificado de Defunción provincial.

Esta propuesta fue aprobada por los participantes del Seminario, considerándosela de gran importancia para el mejoramiento de la vigilancia epidemiológica, y por consiguiente, para la implementación de las políticas sanitarias.

Cabe destacar en este punto las intensas negociaciones que debieron llevarse a cabo con el nivel central nacional, es decir con la Dirección Nacional de Estadísticas de Salud, la cuál por diversos motivos fue reticente a la implementación de las modificaciones propuestas. Ante estas circunstancias las autoridades de salud provincial, debieron finalmente, hacer uso de su potestad y autonomía, para implementar los cambios en el Certificado de Defunción provincial que había consensuado y recomendado el Semina- 


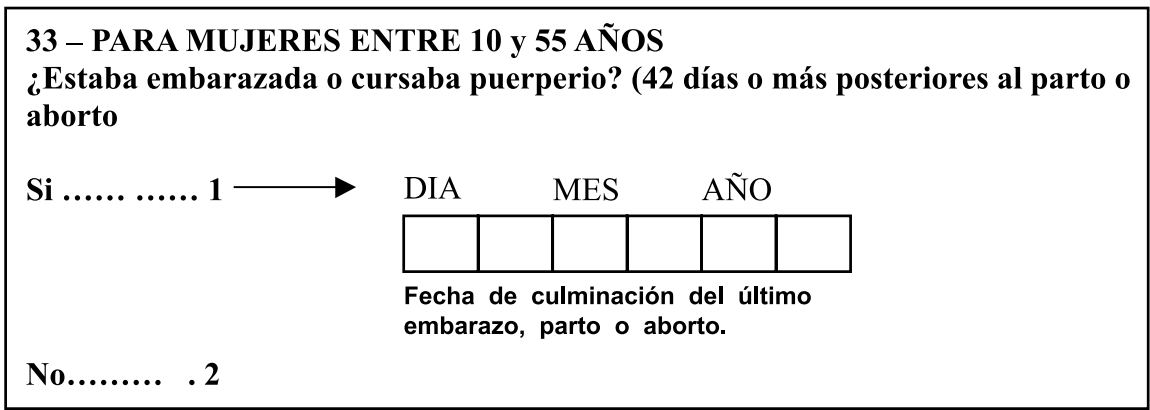

Figura 2 - ITEM 33 del Certificado de Defunción de la Provincia de Santa Fe Figure 2 - ITEM 33 - Death Certificate of Santa Fe State

rio de Rosario. El nuevo certificado se puso en funcionamiento por Resolución Ministerial a partir del 1ro. de Enero de 1995.

\section{Período de estudio}

El nuevo formulario del "Certificado de Defunción" con el "ITEM33" incluido, se puso en funcionamiento en la provincia de Santa Fe a partir del 1ro. de Enero del año 1995, el cuál siguió utilizándose hasta el año 2000 inclusive. Siete años después, en el año 2001, las autoridades nacionales implementan a nivel nacional un nuevo Certificado de Defunción, el cuál requiere un número mucho mayor de información en diversos rubros, incorporando además el Ítem sobre Mortalidad Materna, ya implementado por la provincia de Santa Fe.

Esto significa, que el "ITEM 33" funciona desde el año 1995 y hasta la actualidad. Por lo cuál creí oportuno y necesario el estudio de los resultados de su implementación dado el período considerable (siete años) que lleva su incorporación al Certificado de Defunción, y de esta forma analizar su utilidad como estrategia para eliminar/disminuir el subregistro de la MM.
En este trabajo se analiza un lapso de 14 años (1988 - 2001), comparando un primer período de siete años (1988 - 1994), el cuál no contaba con el "ITEM 33", con el período inmediato posterior también de siete años (19952001) período en el cual el Certificado de Defunción de la Provincia de Santa Fe ya contaba con el mencionado Item. De esta manera se logra el estudio de un indicador de baja frecuencia, en un período relativamente amplio.

\section{Resultados}

Como ya se expusiera, los datos referentes a la MM del período 1980 - 1994 (pre "ITEM 33 ”), muestran una tendencia decreciente, tanto en cifras absolutas cuanto en su indicador.

Sin embargo, una primera observación, ante la introducción del "ITEM 33", muestra que ya en su primer año de implementación, la Muertes Maternas y su indicador experimentaron un incremento que llevó de 18 a 24 el número total de MM (33 \% de aumento), y de 32 a 42 la RMM. Este incremento se consolidó en el período 1995-2001 con una tendencia (variación en el tiempo) en aumento, según los datos publicados hasta la actualidad (Tabla 1).

Tabla 1 - Evolución de la MM Período 1988-2001 - Implementación del “ITEM 33” año 1995 - N y Razones por 100.000 NV

Tabla 1 - MM Evolution - 1988-2001 - "ITEM 33"Implementation - 1995 - Ny Ratios for 100.000 alive newborns

\begin{tabular}{lllllllllllllll}
\hline AÑO & 88 & 89 & 90 & 91 & 92 & 93 & 94 & 95 & 96 & 97 & 98 & 99 & 00 & 01 \\
\hline $\mathrm{N}$ & 17 & 24 & 25 & 19 & 15 & 18 & 18 & 24 & 31 & 31 & 24 & 20 & 25 & 29 \\
Razón & 30 & 43 & 43 & 32 & 26 & 32 & 32 & 42 & 53 & 58 & 46 & 36 & 44 & 57 \\
\hline
\end{tabular}

Fuente: Dirección General de Estadística - Ministerio de Salud Prov. de Santa Fe/ Source: Statistic Gral Direction - Health Ministry of Santa Fe State 
El incremento observado en el segundo período respecto del primero fue del $26 \%$ del mismo, en números absolutos (136 muertes maternas primer período versus 184 muertes maternas en el segundo período), lo cuál significa un aumento del $41.2 \%$ de su indicador la RMM (primer período RMM 34, segundo período RMM 48, siempre por cien mil nacidos vivos)

En el Gráfico 2 se pueden observar las Razones de MM en el período 1988-2001. En el mismo se diferencian los dos períodos analizados, iguales en duración de años, con el fin de hacerlos comparables. Es decir se diferencian dos períodos de siete años cada uno, pre (1988-1994) y pos (1995-2001) implementación del “ÍTEM 33”. En este gráfico se pueden observar además de los valores de las RMM, su tendencia en aumento en el último período, rasgo que la diferencian claramente del período anterior en estudio.

Una segunda observación, es la diferencia en la estructura de la mortalidad materna, es decir en la distribución de las muertes respecto a su incidencia según grupos etáreos
(Tabla 2). Lo que se tradujo en un aumento porcentual de las razones de MM en los extremos de la vida reproductiva, a diferencia de lo observado en años anteriores, obteniéndose la típica curva en "J" que describe la bibliografía., Por falta de información oficial para el año 1988, el período analizado es 1989-2000, excluyéndose premeditadamente el año 2001, con el fin de analizar períodos iguales en años.

Un tercer hallazgo de interés es que se modificó la frecuencia de presentación de las patologías que originaron la muerte, es decir, de la causa de muerte con mayor frecuencia. Según la publicación “Defunciones Maternas y Tasas pordiez Mil Nacidos Vivos Según Causa de Muerte y Grupos de Edad" del período 1.989 - 2.000, editadas por el Ministerio de Salud de la Prov. de Santa Fe, se puede verificar el aumento de las muertes por aborto. Esta causa se ubica, a diferencia de los años pre "ITEM 33", como primera causa de muerte, lugar que anual e históricamente habían ocupado las muertes por hipertensión arterial en el embarazo. Tabla 3.

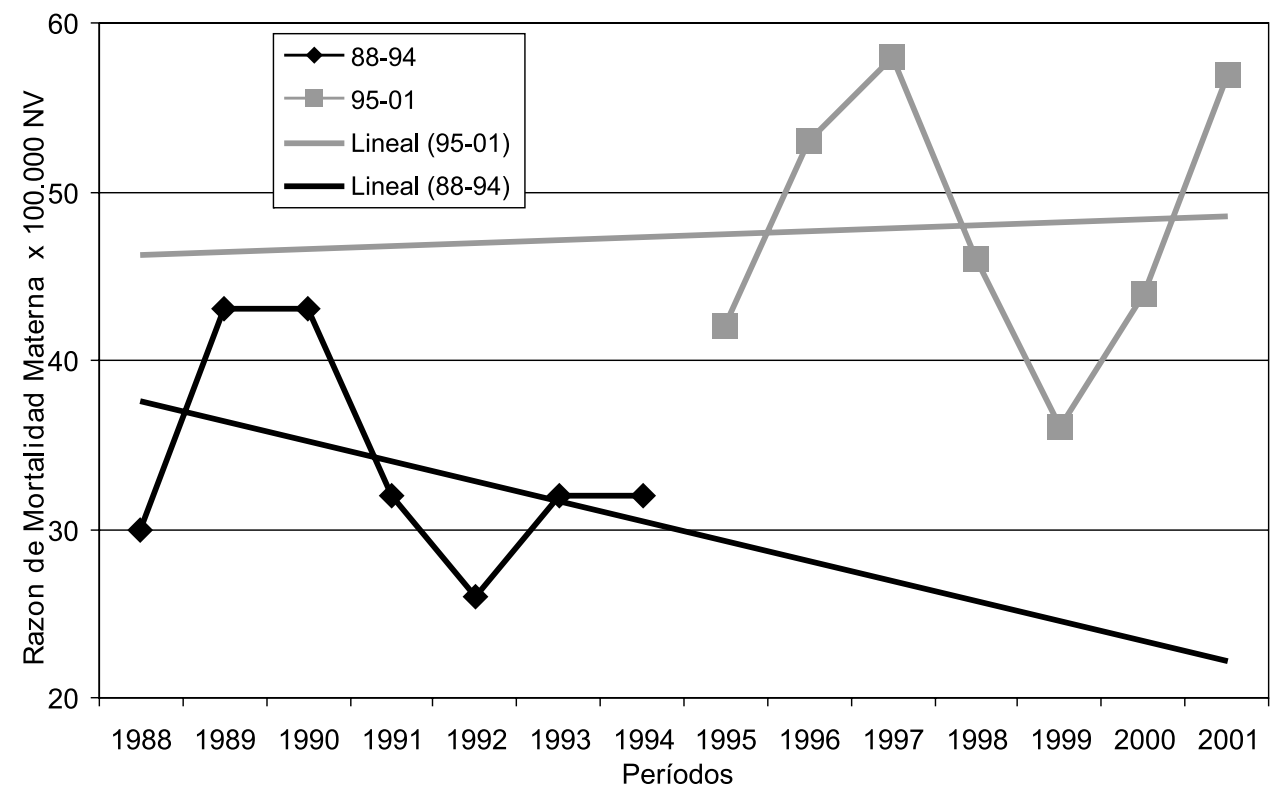

Fuente: Dirección General de Estadística - Ministerio de Salud Prov. de Santa Fe

Source: Statistic Gral Direction - Health Ministry of Santa Fe State

Gráfico 2 - Comportamiento de la Razón de Mortalidad Materna Período 1988-1994 y período 1995-2001

Gráfico 2 - Maternal Mortality Ratio Behavior - 1988-1994 and 1995-2001 periods 
Tabla 2 - Número de Muertes Maternas según Grupo Etáreo -Período 89-94 y período 95-00. (*) Razón por 100.000

Table 2 - Maternal Death Number by age group - 89-94 and 95-00 Periods. ( $\left.{ }^{*}\right)$ Ratio for 100.000

\begin{tabular}{|c|c|c|c|c|}
\hline \multirow{3}{*}{ Grupo Etáreo } & \multicolumn{4}{|c|}{ Período } \\
\hline & \multicolumn{2}{|c|}{ 89-94 } & \multicolumn{2}{|c|}{$95-00$} \\
\hline & $\mathrm{N}$ & Razón (*) & $\mathrm{N}$ & Razón $\left(^{*}\right)$ \\
\hline$<20$ & 15 & 41.9 & 20 & 80.5 \\
\hline $20-34$ & 66 & 26.3 & 94 & 41.6 \\
\hline $35-49$ & 37 & 71.8 & 41 & 164.5 \\
\hline$>49$ & 1 & 926.6 & - & - \\
\hline
\end{tabular}

Fuente: Dirección General de Estadística - Ministerio de Salud Prov. de Santa Fe

Source: Statistic Gral Direction - Health Ministry of Santa Fe State

Tabla 3 - Número de Muertes Maternas Según Causa - Período 89-94 y período 95-00.

Table 3 - Maternal death Number by Cause - 89-94 and 95-00 periods.

\begin{tabular}{cccccc}
\hline \multicolumn{2}{c}{ ABORTO } & \multicolumn{2}{c}{ HEMORRAGIA } & \multicolumn{2}{c}{ HIPERTENSIÓN } \\
Período & \multicolumn{2}{c}{ Período } & \multicolumn{2}{c}{} \\
\hline $89-94$ & $95-00$ & $89-94$ & $95-00$ & $89-94$ & $95-00$ \\
23 & 32 & 12 & 12 & 24 & 21 \\
\hline
\end{tabular}

Fuente: Dirección General de Estadística - Ministerio de Salud Prov. de Santa Fe

Source: Statistic Gral Direction - Health Ministry of Santa Fe State

\section{Discusión}

El Certificado de Defunción tiene relativo valor en cuanto a la estadística médica, ya que no reúne todos los elementos necesarios para que así lo sea. Además sería imposible exigir una completa información en un certificado que el médico realiza en el momento del fallecimiento para que se proceda a inhumar a la muerta, sin que se puedan requerir los datos hospitalarios completos, incluida la autopsia. La estadística y la investigación deben hacerse a partir de instrumentos más completos y con mayor tiempo para obtener toda la información .

En nuestra opinión, los datos provenientes de la RMM pueden mejorarse notablemente, al modificar el Certificado de Defunción incorporándole un apartado que interrogue sobre las muertes maternas. Teniendo en cuenta, además, que tanto la República Argentina como otros la mayoría de los países, utilizan ese documento estadístico para identificar las muertes maternas.

\section{Otros métodos para mejorar la información}

Como ya se ha mencionado, en la mayoría de los países, la información oficial de las muertes maternas tiene un importante subregistro y ello es más evidente en países en vías de desarrollo y especialmente en aquellos en que el aborto no está legalizado.

Las fuentes de información suelen ser múltiples, pero hasta ahora, no existe un modelo universal que satisfaga las necesidades de las diferentes comunidades. En general la primera y básica fuente de datos son las Estadísticas Vitales, publicadas por los respectivos organismos gubernamentales (en general las Direcciones de Estadísticas de los Ministerios de Salud tanto nacional como provinciales), las cuales recogen la información de los Certificados de Defunción vía los Registros Civiles. Sin embargo, frente a la problemática del subregistro, han sido propuestos otros métodos con el fin de mejorar la identificación de las Muertes Maternas. 
El segundo método más habitualmente usado es la revisión de los Certificados de Defunción de mujeres en edad reproductiva.

Un tercer método denominado "Linckage System" ${ }^{23}$, que consiste en el entrecruzamiento de datos del Certificado de Defunción de mujeres en edad reproductiva con los Certificados de Nacido Vivo y de Feto Muerto, método que seguramente puede identificar alguna muerte materna adicional.

Una cuarta fuente de información, son los informes de Autopsias ${ }^{24}$, las cuales no son obligatorias en la mayoría de los países en desarrollo, por lo que este método es ineficaz. Una quinta fuente, la constituye la Revisión de Historias Clínicas de un nosocomio, de las muertes ocurridas a mujeres en edad reproductiva.

La sexta forma en que se ha intentado develar si la muerte fue materna, es la entrevista a médicos, enfermeras y a familiares de la fallecida, que pueden agregar información adicional de interés. En algunos países industrializados (Australia y Nueva Zelanda), utilizan el llamado "Confidential Enquiry into Maternal Death" 25 , cuyo objetivo es recibir opiniones, garantizando estrictas reservas. Mientras que países en vías de desarrollo, utilizan con relativa frecuencia y éxito, el método indirecto denominado "Sisterhood"26,27, que consiste en interrogar a la hermana o hermano de la fallecida, sobre la posibilidad de embarazo de la fallecida, en el momento en que sucedió la muerte.

Por último, el método que este trabajo presenta, es aquel que modifica el Certificado de Defunción, incorporando al mismo un apartado específico para la muerte de mujeres en edad reproductiva.

\section{Confiabilidad de los métodos de identificación}

La poca confiabilidad de los datos surgidos de las Estadísticas Vitales debe ser suplementada por otros métodos. La revisión de los Certificados de Defunción (de mujeres en edad fértil) es un procedimiento fácil y de bajo costo, aunque puede tener errores. El procedimiento “Linckage System” (en- trecruzamiento de Certificados de Defunción de mujeres en edad fértil con Certificados de Nacidos vivos y de Feto Muerto) solo puede detectar alrededor de las $2 / 3$ partes de las muertes maternas, ya que no detecta muertes en gestaciones menores de 20-22 semanas. La revisión de los informes de autopsia, la revisión de las historias clínicas de un nosocomio, las entrevistas a médicos, enfermeras y familiares, pueden también disminuir el error de la identificación. En cualquiera de los casos un Comité de Auditoria, facilitaría la información, pero las dificultades son reales, ya que: hay pocas autopsias, los registros médicos son difíciles de localizar, los investigadores tienen dificultades para acceder a los registros, y los médicos piensan en los posibles problemas legales, que puede acarrear una entrevista.

El método "Confidential Enquiry into Maternal Deaths" es utilizado en países desarrollados (Inglaterra, Australia, Nueva Zelanda, etc,) y se considera que puede brindar una completa información, si se garantiza la reserva. Mientras que el método "Sisterhood" es barato, rápido y fácil de administrar, tiene su aplicación, fundamentalmente en países en vías de desarrollo, en regiones pequeñas y lugares donde no existen registros habituales.

En la República Argentina la determinación oficial de las Muertes Maternas se realiza a través del Certificado de Defunción, el cual hasta el año 2001 no poseía ninguna especificación respecto de las mismas. Se ha sugerido desde el nivel central, es decir, desde el Ministerio de Salud Nacional, la conformación de Comités de Estudios para la Mortalidad Materna, organizados a nivel local, regional, provincial y/o nacional. El objetivo de estos Comités, es la vigilancia epidemiológica de esta causa de muerte, lo que implica la búsqueda, el análisis y las recomendaciones con el fin de lograr un indicador con cifras aceptables. A tales efectos se ha elaborado y publicado en el año 1998, la propuesta normativa nacional "VIGILANCIA Y CONTROL DE LA MORTALIDAD MATERNA"28.

El método adoptado por la Provincia de 
Santa Fe, que analiza este trabajo ha sido implementado en pocos lugares del mundo, y son aún más escasos los reportes que informan sobre su impacto en la mejora de la calidad del indicador.

La inclusión del ITEM 33 en el Certificado de Defunción, ha revertido la tendencia descendente de los registros de las muertes maternas en la provincia de Santa Fe, por lo que en mi opinión, esta intervención ha contribuido notablemente, como aquí se demuestra, a disminuir el subregistro de las muertes maternas.

Otro aspecto que consideramos importante mencionar es el comportamiento en el período estudiado, de la TMI en comparación con el descrito por la RMM, ya que ambos se aceptan como óptimos indicadores de las condiciones de vida y de salud de una población, como considera Victora y col. en su trabajo "Epidemiología de la Desigualdad" ${ }^{29}$.

En el año 1990 la TMI se ubicaba en 23,5 defunciones por mil nacidos vivos, mientras que para el año 2001, se ubicó en 14.1 defunciones por mil nacidos vivos ${ }^{30}$, presentando una tendencia claramente descendente en la última década del Siglo XX, contrariamente a lo ocurrido con la Razón de Mortalidad Materna. Esta aparente contradicción en el comportamiento de ambos indicadores, estaría señalando, el mejoramiento de los registros de las Muertes Maternas y no un aumento real de la incidencia de esta causa de muerte.

Otro hecho importante de señalar, es que a partir de la introducción del "ITEM 33", la causa más frecuente de muerte materna es el aborto, pese a que una jurisdicción tan importante numéricamente como la de Rosario ha implementado recientemente en el ámbito municipal un programa de procreación responsable, si bien se sabe que programas de orden poblacional, donde intervienen factores sectoriales y extra-sectoriales, suelen demandar años para observar resultados esperados, teniendo en cuenta además la doble administración en salud que rige en dicha ciudad.

Los resultados hallados confirman los datos señalados en la literatura, tanto nacional como internacional, ya mencionada en este trabajo, respecto del subregistro de la mortalidad materna.

Los hallazgos de este trabajo, deberían ser interpretados teniendo en cuenta el contexto en el cuál se llevó a cabo el mismo. Es decir teniendo en cuenta que la provincia de Santa Fe es la segunda en importancia de la República Argentina, con escasa población rural, que el $97 \%$ de los partos son institucionales, y que la investigación se llevó a cabo en el marco de la implementación de un programa materno infantil. Pese a lo cuál la cifra atribuida al subregistro en este estudio, llegaría al $41 \%$, coincidiendo con investigaciones previas.

\section{Conclusiones}

El “Programa Provincial MaternoPerinatal”, puesto en marcha en el año 1994 normatizó oficialmente, por primera vez para toda la provincia de Santa Fe, las acciones para la atención materno perinatal. Este programa contó entre sus objetivos, el de solucionar el problema del subregistro de la Mortalidad Materna.

De los resultados obtenidos en la presente investigación, se puede considerar que la Mortalidad Materna es un problema innegable para la provincia de Santa Fe, y que cada una de sus regiones sanitarias plantea un problema diferente a resolver.

Por lo cual es impostergable la implementación de un sistema de "inteligencia epidemiológica” para las mujeres en edad reproductiva

La introducción del “ITEM 33” en el Certificado de Defunción de la Provincia de Santa Fe produjo modificaciones importantes en los datos sobre MM. Estas modificaciones pueden ser atribuidas a la disminución del subregistro, ya que no se encuentran razones valederas para pensar en un aumento real en la ocurrencia de muertes maternas, teniendo en cuenta, que el número de nacidos vivos (denominador de la RMM) no se ha modificado bruscamente en el período de estudio, y que la TMI ha presentado una 
tendencia decreciente en el último decenio.

Por lo cual se concluye que: este instrumento de recolección de datos permitiría corregir el subregistro y obtener datos de mayor calidad que, en consecuencia, posibiliten la implementación de políticas y programas de salud basados en datos que reflejen la verdadera situación sanitaria.

\section{Agradecimiento}

El autor agradece la valiosa colaboración, las sugerencias y comentarios de los siguientes profesionales: Dra. María del Carmen Troncoso $(\dagger)$ ex Directora de la Carrera de Epidemiología del Instituto de la Salud "Juan
Lazarte" de Rosario, Dr. Guillermo Carrolli Director del Centro Rosarino de Estudios Perinatales (CREP), Lic. Alicia Aronna Directora del Sistema Municipal de Estadísticas para la Salud de Rosario, Dr. Ernesto Taboada, Director de la Carrera de Epidemiología del Instituto de la Salud "Juan Lazarte" de Rosario. Como así mismo a los integrantes de la Dirección General de Estadísticas del Ministerio de Salud de la Provincia de Santa Fe, en la persona de su Directora Lic. María Rosa López Facciano y a mi Maestra y Amiga Dra. Rita Barradas Barata del Departamento de Medicina Social de la Facultad de Ciencias Médicas Santa Casa de São Paulo Brasil.

\section{Referências}

1. Organización Pan Americana de la Salud. Programa Regional de Salud Materno-Infantil. Fascículo IV. Serie Mortalidad Materna b y c. Washington (DC); 1989.

2. Fathalla M, Rosenfield A, Indriso C, Sen D, Artnam S. Mortalidad Materna. En: Manual sobre reproducción humana, FIGO 1993; Vol. 3, p. 85-104.

3. Benedetti JT, Starzyk P, Frost F. Maternal death in Washington state. Obstet Gynecol 1985; 66(1): 99-101.

4. Allen M, Chavkin W, Marinoff J. Ascertainment of Maternal Death in New York City. Am J Public Health 1991; 81(3): 380-2.

5. Puffer R, Griffith G. Características de la mortalidad urbana. Washington (DC) Organización Pan Americana de la Salud (OPS - Publicación Científica 151).

6. Ni-H, Rossignol AM. Maternal Death among Woman with Pregnancies outsiders of Family Planning in SiChina. China Epidemiology 1994; 5(5); 490-4.

7. Organización Pan Americana de la Salud. Boletín Epidemiológico 1993; 14(1); 1-9.

8. Organización Pan Americana de la Salud. XLII Reunión del Comité Regional de la OMS para las Américas. Washington DC, 1990. Documento OPS-OMS. CSP, 2310 (ESP) 20 de Julio de 1990.

9. Laurenti R. Maternal mortality in Latin American urban areas: the case of Sao Paulo, Brazil. Bull Pan Am Health Organ 1993; 205-14.

10. 26va Conferencia Sanitaria Panamericana. Estrategia regional para la Reducción de la Mortalidad y Morbilidad Maternas. Washington (DC); 2002.
11. Ministerio de Salud y Acción Social de la República Argentina. La Mortalidad Materna en la República Argentina. Programa Nacional de Estadísticas de Salud.. Serie 8. Número 4. Junio de 1987.

12. Ministerio de Salud Pública de la Prov. de Córdoba. Departamento de Estadísticas de Salud. Año 1991.

13. Bloch C, Torres de Quinteros ZC, et al. El proceso salud enfermedad en el primer año de vida. Estudio de una cohorte, Rosario (Argentina) 1981-82. primera parte. Cuadernos Médico-Sociales 1985; 32: 5-19.

14. Bloch C, Torres de Quinteros ZC, et al El proceso salud enfermedad en el primer año de vida. Estudio de una cohorte, Rosario (Argentina) 1981-82. Segunda parte. Cuadernos Médico-Sociales 1985; 32: 25-34.

15. Faúndes A, Cecatti JG. Morte materna uma tragedia evitable. UNICAMP; 1991. p. 22.

16. World Health Organization (WHO), UNICEF. Revised 1990 Estimates of maternal mortality: WHO/FRH/ MSM/96.11. Geneva: WHO, 1996.

17. World Health Organization (WHO) and United Nations Development Programme Stengthening National Capacity to Reduce Maternal Death and Disabilities. Center for Population and Family Health Columbia University, March 1994.

18. Ibidem No 11 .

19. Ibidem No 12 .

20. Programa Nacional de Estadísticas de Salud. Estadísticas de Salud. Serie 5. No 23-40. Dirección de Estadísticas. Ministerio de Salud de la Nación, Buenos Aires, Argentina. 
21. Vinocur J y col. La mortalidad materna por regiones geográficas - República Argentina - Años 1990-1993. Programa Nacional de Estadísticas de Salud. Publicación No 27 Serie 8. Ministerio de Salud Nacional. Buenos Aires, Argentina; 1996.

22. Torres de Quinteros ZC y col. Mortalidad de mujeres en edad reproductiva (Una aproximación desde los Certificados de Defunción) Investigación en Salud, Publicación Científica de la Secretaría de Salud Pública Municipal. Vol. 3. No 1 y 2. Rosario; 2000.

23. Jocumbs S, Mitchel E, Entman S, Piper J. Monitoring Maternal Mortality Using Vital Records Linkage. Am J Prev Med 1995; 11(2): 132-133.

24. Rizzi R, Ruiz Córdoba R, Mguna J. Maternal Mortality due to Violencie. Int. J Gynec \& Obstet 199863 (Suppl. 1): S19-S24.

25. Centro Rosario de Estudios Perinatales - UNICEF Argentina Desarrollo de un Modelo para la Auditoria de Mortalidad Materna en Argentina. Informe Final; 1997.
26. Hernández B, Chirinos J, Romero M, Langer A. Estimating Maternal Mortality in Rural areas of Mexico: The application of an Indirect Demographic Method. Int J Gynecol Obstet. 1994; 46(3): 285-289.

27. Walraven G, Mkanje R, Van Roosmalen J, Van Dongen P, Dolmans W. Assessment Mortality in Tanzania. Br. J Obstet Gynacol 1994; 101(5): 414-417.

28. Elizalde E y otros Vigilancia y Control de la Mortalidad Materna (M.S.Y.A.S.) Arte Gráfica PAPIRO; 1992.

29. Victora C, Barros F, Vaughan J. Epidemilogía de la Desigualdad. Serie PALTEX No 27 OPS/OMS; 1992.

30. Ministerio de Salud de la Prov. de Santa Fe Estadísticas Vitales Año 1990 1994. Dirección General de Estadística; 1995. Santa Fe, Argentina.

recebido em: 20/04/2004

versão final apresentada em: 08/11/2004

aprovado em: 17/11/2004 